\title{
OCORRÊNCIA DE FUNGOSEM LEITE CRU PROVENIENTE DE TANQUESDE REFRIGERAÇÃO E LATÕES DE PROPRIEDADES LEITEIRAS, BEM COMO DE LEITE COMERCIALIZADO DIRETAMENTE AO CONSUMIDOR
}

\section{P.A. Melville, M. Ruz-Peres, E. Yokoia, N .R. Benites}

Universidade de São Paulo, Faculdade de Medicina Veterinária e Zootecnia, Departamento de Medicina VeterináriaPreventiva eSaúdeA nimal. Av. Prof. Dr. Orlando Marques dePaiva, 87, CEP:05508-000, São Paulo, SP, Brasil. E-mail: melville@usp.br

\section{RESUMO}

\begin{abstract}
Osfungosfilamentososeleveduras podemestar associados adiferentes patologiasno homem e animais. O leite e seus derivados contaminados com estes mi crorganismos podem constituir potenciais vias detransmissão dezoonoses a eles relacionadas. Foram analisadas 70 amostras de leite, sendo 50 de tanques de refrigeração, 10 de latões de propriedades de exploração leiteira e 10 de latões de distribuidores que comercializam leite informal, visando a comparação da qualidade destes produtos quanto à presença e quantidade de fungos. Foram isolados, em diferentes percentagens, fungosfilamentososelevedurasa partir detodas asamostras deleitedas diferentes procedências: Candida spp. (C. krusei, C. guilliermondii, C. tropical is, C. kefyr, dentreoutras), G eotrichum spp., R hodotorula spp., Trichosporon spp., A ureobasidium spp., Penicillium spp.,A cremonium spp., Chrysosporium spp., M ucor spp. e A spergillus spp. Não houve diferença estatisticamente significante entre as medianas das quantidades de unidades formadoras de colônias de fungos/ $\mathrm{mL}$ de leite das diferentes procedências, indicando que o nível de contaminação por fungos nas amostras destas três origens foi similar. Pode-se verificar que em todas as amostras deleite cru havia a presença defungos, todos passíveis agentes de micoses oportunísticas.
\end{abstract}

PALAVRAS-CHAVE: Leite cru, leveduras, fungos filamentosos.

\section{ABSTRACT}

OCCURRENCE OF FUNGI IN RAW MILK FROM BULK TANKS, MILK CANS OF DAIRY FARMSAND MILK SOLD DIRECTLY TO THE CONSUMER. Filamentous fungi and yeasts can be associated to various pathologies in man and animals. It must beconsidered that milk and dairy products when contaminated with these microorganisms can represent a potential means of zoonosistransmission. In the present study, 70 samples of raw milk wereanalysed, 50 from bulk tanks, 10 from milk cansfrom dairy farms, and 10 from cans of distributorsthat storeand sell milk sold on the informal (unregulated) market, comparing the quality of these products as to the presenceand quantity of fungi. M oulds and yeasts wereisolated in different percentages from all of the samples of the different sources: Candida spp. (C . krusei, C . guilliermondii, C. tropicalis, C. kefyr etc.), Geotrichum spp., Rhodotorula spp., Trichosporon spp., A ureobasidium spp., Penicillium spp., A cremonium spp., Chrysosporium spp., M ucor spp. and A spergillus spp. No statistically significant differencewas observed between medians of thequantity of col onies forming units of fungi/ $\mathrm{mL}$ of milk from the different sources, indicating that thelevel of contamination by fungi was similar when the threesources were compared. Thepresence of fungi was observed in all samples of raw milk, all these microorganisms able to cause opportunistic mycoses.

KEY WORDS: Raw milk, yeasts, filamentous fungi.

\section{INTRODUÇÃO}

Os fungos são microrganismos comuns na natureza, estando presentes em ambientes aquáticos, terrestres e no ar. O desenvolvimento destes microrganismos podedeterminar doenças infecciosas ou tóxicas, em vegetais e animais, incluindo o homem. A simples presença do fungo não éfator determinante de doença, a qual depende da relação parasita/ hospedeiro (LACAZ et al ., 2002).

Osreservatórioshabituais dosfungosqueinfectam o homem eo animal podem ser o próprio homem eo animal, ou um sítio na natureza, onde o fungo se desenvolvecomosaprófita. Asmicoses são classifica- 
das como: (1) micoses superficiais, que se localizam nas camadas mais superficiais da pele ou dos pelos edesencadeiamumarespostainflamatória,(2)micoses cutâneas, asquaisselocalizamnapele, pelo ou unhas emucosasemmaior extensão, (3) micoses subcutâneas, encontradas na pele e tecidos subcutâneos, (4) micoses sistêmicas, queatingem os órgãos internose vísceras, podendo abranger muitos tecidos e órgãos diferentes (Trabulsi et al., 1999). Dentreas manifestaçõesclínicasemanimaisassociadasaosfungospodese citar a mastite micótica.

A presençadefungosnoleitepodeestar associada à ocorrência de casos de mastite infecciosa no rebanho, ou podeestar relacionadaao nível dehigieneda ordenha e do ambiente (CousINS \& BRAMLEY, 1981).

A contaminação do leite por mi crorganismos indesejáveis, como os fungos, pode causar alterações físico-químicasno mesmo, limitando suadurabilidadeedeseus derivados, além dedeterminar problemas econômicos e de saúde pública (ANDRADE, 2001).

$\mathrm{O}$ tratamento térmico do leite (pasteurização ou fervura) permitea redução da ocorrência dedoenças causadas por microrganismos, veiculadas pelo leite eseusderivados. Entretanto, torna-seimportanteatentar para o risco queconstitui a presençad defungos no leite, principalmenteconsiderando-seque, em diversaslocalidades, écomumo hábito do consumodeleite e produtos lácteos crus. O leite cru, muitas vezes é comercial izado nas periferias das cidades, constituindo o chamado "leiteinformal ou clandestino", não submetido a qual quer tipo de tratamento para redução desua carga microbiana eeliminação dos principais patógenos.

Segundo QueIROz (1995), embora exista a proibição legal imposta à comercialização do leite cru no Brasil (Lei n 1.283 de 18/ 12/ 1950 e Decreto número 30.691 de29/ 03/ 1952), avendadestetipo deleitetem sido amplamentereal izada na periferia daCidadede São Paulo, eem diversas cidades do interior, muitas das quais com elevado nível socioeconômico ecultural. Em Minas Gerais, estima-se que $47 \%$ do leite consumido pela população seja proveniente da comercialização clandestina do leite cru (Brandão, 1994).

$\mathrm{Na}$ Comunidade Européia, as políticas que estabelecem as diretrizes para o comércio de leite "in natura" variam consideravelmente entre os países, sendo que, na maioria, este comércio é oficialmente proibido. A Inglaterraéumadas exceções, nãopossui normasqueproíbamocomércio destetipo deproduto, sendoainspeção real izada na propriedadedeorigem através do controledaqualidadedoleitenostanques de refrigeração e do monitoramento periódico da sanidade do rebanho. Em 1997 foi realizada uma pesquisa neste país, onde foram analisadas 1.097 amostras de leite cru comercializadas legalmente, oriundas de 242 estabelecimentos comerciais, tendoseverificado que 41 amostras de 21 pontos devenda estavam contami nadas com microrganismos potencialmente patogênicos. Nesta mesma pesquisa, observou-se também que a frequência de doenças relacionadas ao consumo deleite "in natura" émaior nos países onde sua comercialização é permitida, demonstrando que a real ização da aval iação da qual idade higiênica do leite e o monitoramento sanitário do rebanho não constituem garantia da segurança alimentar do produto final (FONSECA \& SANTOS, 2000).

Em cidades do interior deSão Paulo, emesmo nas periferias das grandes cidades ou em locais dedifícil acesso ao produto fiscal izado pela inspeção sanitária, existeum hábito comum da ingestão deprodutos adquiridos diretamente do produtor, como ovos e frangos caipiras, frutas, verduras e legumes, queijo fresco, leite cru etc, alimentos que são produzidos pelos própriosproprietários, envolvendoumnúmero muito pequeno de funcionários, geralmente sem maior experiência técnica.

Assim sendo, deve-se atentar para a presença de microrganismos, dentre os quais os fungos filamentososeleveduras noleite, o quepoderepresentar potencial risco à saúde humana.

O presente trabal ho foi delineado considerandosea importância e rel evância destegrupo de microrganismos, fungosfilamentososeleveduras, tendo em vista sua participação como agentes de importantes patologias muitas das quais com caráter zoonótico. Deve-seconsiderar ainda queoleiteeseus derivados contaminadoscomestesmicrorganismospodem constituir potenciais vias de transmissão de zoonoses a eles relacionadas. N estetrabal ho pretendeu-seanalisar amostras deleitecru dostiposA , BeC col hidasnas próprias propriedades leiteiras, bem como amostras deleitecomercializadas diretamenteao consumidor, procedendo-se ao isolamento, identificação e contagem de unidades formadoras de colônias (U.F.C.) de fungos filamentosos e leveduras, visando-se a comparação da qualidade destes produtos quanto à presença de fungos.

\section{MATERIAL E MÉTODOS}

As propriedades envolvidas nesta investigação localizam-se essencialmente nas regiões de Campinas, Itu ePirassununga, todas pertencentes aoEstado de São Paulo, Brasil. Foram avaliadas amostras de leiteoriundas de50tanques derefrigeração quearmazenamleitedepropriedades deexploraçãoleiteirade leite cru dos tipos A, B e C, cujo destino final é o processamentoemestabelecimentoindustrial. Foram colhidas, ainda, amostras deleitede10propriedades produtoras de leite cru dos tipos $\mathrm{B}$ e $\mathrm{C}$, as quais 
armazenamo leiteem latões (vasilhames com $50 \mathrm{~L}$ de capacidade) equeparticipam efetivamentedomercado deleite "informal ou clandestino".

Além das amostras de leite obtidas diretamente das propriedades, também foram examinadas amostras provenientes de 10 latões de transportadores e distribuidores (automóveis do tipo perua) quearmazenamecomercializamdiretamenteao consumidor o leitecru obtido das propriedades produtoras deleite tipo C.

Cada amostra de leite (volume de aproximadamente $100 \mathrm{~mL}$ ) foi col hida em frasco devidro esterilizado e transportada sob refrigeração $\left(2\right.$ a $\left.8^{\circ} \mathrm{C}\right)$ ao laboratório onde foram preparadas diluições seriadas da mesma em solução fisiológica ( $0,85 \%)$ esterilizada.

A partir decadadiluiçãofoi colhidaumaalíquota de $0,1 \mathrm{~mL}$ para cultivo (utilizando-se a técnica de spread plate ou, espal hamento em superfície) em duplicata, tanto em ágar Sabouraud-dextrose com cloranfenicol (100 mg/ L) como em ágar batatadextroseacidificado com solução esterilizada deácido tartárico a 10\% (Araújo et al., 2001).

Para a pesquisa deCryptococcus neofor mans, procedeu-seà semeadura da amostra de leite $(0,1 \mathrm{~mL}$ utilizando-sea técnica despread plate) em meio decultura seletivoágar Niger (LARONE, 1995). Paraidentificação das espécies de $C$ andida o meio sel etivo ediferencial utilizado foi o ágar Pagano-Levine (CRUZ, 1985), também setendo real izado asemeadurade $0,1 \mathrm{~mL}$ deleite utilizando-se a técnica de spread plate.

Todos estes meios foram incubados a $25^{\circ} \mathrm{C}$ em estufamicrobiológica, por umperíodomínimo desete dias, visando o isol amento, identificação econtagem de unidades formadoras de colônias de fungos filamentosos eleveduras/ $\mathrm{mL}$.

As leveduras foram identificadas de acordo com LodDer (1970) e KREEGER-VAN-RIG (1984) e os fungos filamentosos foram identificados de acordo com a chave deBarnett \& Hunter (1972) e Von Arx (1974), utilizando-se a descrição das características macroscópicas emicroscópicas.

A anál ise estatística foi realizada utilizando-se o "software". Foramutilizadososseguintestestesestatísticos: Fischer, Dunn e Kruskal-Wallis.

\section{RESULTADOS}

Deumtotal de50amostras deleitecru provenientes de tanques de refrigeração de propriedades de exploração leiteira, foram isolados os seguintes fungos filamentosos e leveduras: A ureobasidium spp. Candida spp. (C. krusei, C. parapsilosis, C. kefyr, C. albicans, C. guilliermondii, C. Iusitaniae, C. tropicalis), R hodotorula spp., Geotrichum spp., Trichosporon spp.,
M ucor spp., A spergillus spp., Chrysosporium spp., A cremonium spp., Penicillium spp. (Tabela 1). As freqüências deisolamentos deC . krusei (70\%), C. tropical is (54\%), C. guilliermondii (52\%), C. kefyr (34\%), Geotrichum spp. (52\%) e R hodotorulaspp. (40\%), foramestatisticamentemaiores $(p<0,05)$ quando comparadas com as dos demais fungos isolados (Tabela 1). Observou-se a presença de fungos em todas as amostras de leite oriundas de tanques de refrigeração.

Deumtotal de10amostras deleiteprovenientes de latões de propriedades leiteiras, foram isolados os seguintes fungos:C andida spp.(C. krusei, C. parapsilosis, C. kefyr, C. guilliermondii, C. Iusitaniae, C. tropicalis), Rhodotorula spp., Geotrichum spp., M ucor spp., Chrysosporium spp., Trichosporon spp. e Penicillium spp. (Tabela 1). Observou-sea presençadefungosem todas as amostras de leite oriundas de latões de propriedades de exploração leiteira.

Das 10 amostras de leite oriundas de latões presentesemtransportadoresedistribuidoresquearmazenamecomercializamleiteinformal, foramisolados os seguintes fungos filamentosos e leveduras: A ureobasidium spp., Candida spp. C. krusei, C. parapsilosis, C. albicans, C. guilliermondii, C. tropical is C. kefyr, C. lusitaniae), R hodotorula spp., G eotrichum spp., Trichosporon spp., M ucor spp., A spergillus spp., Chrysosporium spp., Penicillium spp. Observou-se a presença de fungos em todas as amostras de leite oriundas delatõesdostransportadoresedistribuidores de leite informal.

Considerando-seas50amostras deleiteoriundas detanques derefrigeração, a mediana das contagens deU.F.C. defungos/ $\mathrm{mL}$ foi de $28,5 \times 10^{2} / \mathrm{mL}(0,16 a$ $332,50 \times 10^{2} / \mathrm{mL}$ ) deleite. Em relação as 10 amostras deleiteoriundas delatões depropriedades deexploração, a mediana das contagens deU.F.C. defungos/ $\mathrm{mL}$ foi de $12,91 \times 10^{2} / \mathrm{mL}\left(0,22-138,66 \times 10^{2} / \mathrm{mL}\right)$ de leite. Com relação as 10 amostras deleiteoriundas de latões presentes em transportadores edistribuidores que armazenam e comercializam leite informal, a medianadascontagensdeU.F.C. defungos/ $\mathrm{mL}$ foi de $14,07 \times 10^{2} / \mathrm{mL}\left(2,2-78 \times 10^{2} / \mathrm{mL}\right)$ de leite.

Das 70 amostras deleitedediferentes procedências analisadas, as leveduras estiveram presentes em $100 \%$ delas. Por sua vez, os fungos filamentosos foram isol ados de $26 \%$ das amostras de tanque, $60 \%$ das amostras de latões de distribuidores e 30\% de latões de propriedades leiteiras.

Quando comparadasas medianas entreasquantidades deunidades formadoras decolônias defungos/ $\mathrm{mL}$ de leite entre as amostras colhidas de tanques derefrigeração $\left(28,5 \times 10^{2}\right.$ U.F.C./ mL), latõesde propriedades de exploração leiteira $\left(12,91 \times 10^{2}\right.$ U.F.C./ $\mathrm{mL}$ ), e latões de distribuidores $\left(14,07 \times 10^{2}\right.$ U.F.C./ $\mathrm{mL}$ ), não houve diferença estatisticamente significante. 
Tabela 1 - Freqüências de isolamentos de leveduras efungos filamentosos em amostras deleitecolhidas de 50 tanques de refrigeração, 10 latões de propriedades leiteiras e 10 latões de transportadores e distribuidores que armazenam e comercializam leite informal.

\begin{tabular}{|c|c|c|c|c|c|c|}
\hline \multirow{3}{*}{$\begin{array}{l}\text { Origem da amostra } \\
\text { Fungos isolados }\end{array}$} & \multicolumn{6}{|c|}{ Origem da amostra } \\
\hline & \multicolumn{2}{|c|}{$\begin{array}{c}\text { Tanque de } \\
\text { refrigeração }(\mathrm{N}=50)\end{array}$} & \multicolumn{2}{|c|}{$\begin{array}{l}\text { Latão de } \\
\text { distribuidor }(\mathrm{N}=10)\end{array}$} & \multicolumn{2}{|c|}{$\begin{array}{l}\text { Latão de propriedade de } \\
\text { exploração leiteira }(\mathrm{N}=10)\end{array}$} \\
\hline & $\bar{N}$ & $\%$ & $\mathrm{~N}$ & $\%$ & $\overline{\mathrm{N}}$ & $\%$ \\
\hline A ureobasidium spp. & 4 & 8 & 1 & 10 & 0 & 0 \\
\hline C. albicans & 2 & 4 & 1 & 10 & 0 & 0 \\
\hline C. krusei & 35 & $70^{*}$ & 6 & 60 & 7 & 70 \\
\hline C. parapsilosis & 15 & 30 & 3 & 30 & 3 & 30 \\
\hline C. guilliermondii & 26 & $52 *$ & 7 & 70 & 7 & 70 \\
\hline C. tropicalis & 27 & $54 *$ & 4 & 40 & 5 & 50 \\
\hline C. kefyr & 17 & $34 *$ & 5 & 50 & 2 & 20 \\
\hline C. Iusitaniae & 9 & 18 & 2 & 20 & 2 & 20 \\
\hline Geotrichum spp. & 26 & $52 *$ & 4 & 40 & 5 & 50 \\
\hline Trichosporon spp. & 4 & 8 & 6 & 60 & 4 & 40 \\
\hline Rhodotorula spp. & 20 & $40^{*}$ & 7 & 70 & 6 & 60 \\
\hline $\begin{array}{l}\text { Presença de Leveduras } \\
\text { nas origens }\end{array}$ & 50 & $100^{* k}$ & 10 & 100 & 10 & $100^{* *}$ \\
\hline Penicillium spp. & 4 & 8 & 1 & 10 & 2 & 20 \\
\hline A cremonium spp. & 5 & 10 & 0 & 0 & 0 & 0 \\
\hline Chrysosporium spp. & 2 & 4 & 4 & 40 & 1 & 10 \\
\hline M ucor spp. & 3 & 6 & 1 & 10 & 1 & 10 \\
\hline A spergillus spp. & 1 & 2 & 1 & 10 & 0 & 0 \\
\hline $\begin{array}{l}\text { Presença de Fungos } \\
\text { filamentosos nas origens }\end{array}$ & 13 & 26 & 6 & 60 & 3 & 30 \\
\hline
\end{tabular}

*A s frequências de isolamentos deC. krusei, C. guilliermondii, C. tropical is, C. kefyr, R hodotorula spp. eG eotrichum spp., em amostras col hidas de tanques de refrigeração, foram estatisticamente mai ores $(p<0,05)$ quando comparadas com aos demais fungos isolados.

**A freqüência de isolamento de leveduras a partir de tanques de refrigeração elatões de propriedades deexploração leiteira foi maior $(p<0,05)$ do que a ocorrência de fungos filamentosos.

\section{DISCUSSÃO}

No presenteestudo, foi isoladaumagrandevariedade de fungos filamentosos e leveduras a partir de todas amostras deleitecol hidas detanques derefrigeração elatões de propriedades leiteiras, bem como de leiteoriundo delatões presentes em transportadorese distribuidores que armazenam e comercializam leite informal.

Todos os microrganismos isolados, tais como A ureobasidium spp., C. albicans, C. krusei, C. parapsilosis, C. guilliermondii, C. tropicalis, C. kefyr, C. Iusitaniae, Geotrichum spp., Trichosporon spp., R hodotorula spp., Penicillium spp., A cremonium spp., Chrysosporium spp., M ucor spp. eA spergillus spp. podem estar associados a quadros de micoses oportunísticas (TrabuLsI et al., 1999), quesão infecçõescausadas por fungosdebaixa virulência, queconvivem pacificamentecomo hospedeiro mas que, ao encontrarem condições favoráveis, desenvolvem seu poder patogênico.
As micoses oportunísticas podem estar associadas a fatores intrínsecos ao hospedeiro (neoplasias, diabetes, dentre outras, bem como todas as doenças que alteram a imunidade celular) ou fatores extrínsecos(antibioticoterapia, corticoidoterapia, etc.) (Trabulsl et al., 1999).

Deformageral estesmicrorganismosestão associadosaquadrospatológicoscomo al ergias, otomicoses, ceratomicoses, lesões do palato duro, meningites, endocardites, doenças sistêmicas, gastro-intestinais, pulmonares, geniturinárias, cutâneas, subcutânease ósseas (KERN \& BLEVINS, 1999).

Assim sendo, pode-se observar que todos os fungosfilamentososelevedurasisoladosdas amostras deleitedas diversas origens no presenteestudo apresentam potencial patogênico, principalmenteseo hospedeiro encontrar-sedebilitado pelos fatores mencionados anteriomente. Destaforma, o consumo de leite contaminado por algum destes agentes, ou mesmo de toxinas por eles produzi- 
das, poderia acarretar danos à saúde do consumidor.

Os dados obtidos no presente estudo permitem observar agrandevariedadedegêneros eespécies de fungos isolados das amostras analisadas, que totalizaram 16 espécies, dentre leveduras e fungos filamentosos.

Todasasespécies delevedurasencontradas, foram isoladasamostrasdetanques derefrigeração, latões de distribuidores deleite informal elatões de propriedadesleiteras, à exceção deC . al bicans, A u reobasidiumspp., A cremonium spp. eA spergillus spp., os quaisnão foram isolados de amostras de latões de propriedades de exploração leiteira, e Acremonium spp. que não foi isolado deamostrasoriundas delatões dedistribuidores de leite informal. A freqüência de isolamento de levedurasapartirdetanquesderefrigeraçãoelatõesde propriedades leiteiras foi maior $(p<0,05)$ do quea de fungos filamentosos.

KLosSOWSKA \& MALINOWSI (2001) realizaram examesmicrobiológicosem 66 amostras deleiteprovenientes detanques der efrigeração dediferentes propriedades de exploração leiteira, tendo isolado, dentre outros microrganismos, C. albicans, C. krusei, C. tropicalis, C. kefyr, Geotrichum spp., Trichosporon spp. eA spergillus spp, todos estes agentes também isolados no presente estudo.

SWINNE et al . (1997) identificaram leveduras em 70 tanques de armazenamento de leite de fazendas na Bélgica, observando maior freqüência de C. kefyr, C. rugosa eP ichia kluyueri. N opresenteestudonão foram isoladas $C$. rugosa ePichia kluyueri.

Segundo H ARIDY (1992)foramisoladas126espécies de leveduras de amostras de leite cru colhidas em fazendas deexploraçãoleitei ranacidadedeEI Minia, norte do Egito, sendo que as mais freqüentes foram Trichospor on beigelii eR hodotorula spp., tambémobservados no presente estudo.

JODRAL etal. (1993) isolaramequantificaramfungos em 64 amostras de leite cru e 33 amostras de leite pasteurizado, sendo que os gêneros de fungos mais freqüentemente isolados foram Geotrichum spp., Fusarium spp., Penicillium spp. e A spergillus spp. no leite cru e os mesmos gêneros de microrganismos, à exceção de G eotrichum spp., em leite pasteurizado. A média da contagem no leitecru foi de230U.F.C./ $\mathrm{mL}$ e noleitepasteurizado foi de25U.F.C./ mL. No presente estudo, também foram isolados estes mesmos microrganismos à exceção de Fusarium spp. e, as medianas das quantidades deU.F.C.s defungos/ $\mathrm{mL}$ foram 28,5 $(0,16$ a 332,50$) \times 10^{2} / \mathrm{mL}, 12,91(0,22-138,66) \times 10^{2} / \mathrm{mL}$ e $14,07\left(2,2\right.$ - 78) $\times 10^{2} / \mathrm{mL}$, respectivamente, nos tanques derefrigeração, latões de propriedades deexploração leiteira e latões de distribuidores, podendo-se observar que os val ores obtidos foram superiores aos verificados por JodRAL et al. (1993).
Mutukumira et al. (1996) analisaram microbiologicamente 10 amostras deleite cru detanques de 34 produtores. A contagem de leveduras e fungos filamentosos foi <100U.F.C./ $\mathrm{mL}$ em 7 das 10 amostras analisadas. Por sua vez, Desmasures et al. (1997) estudaram 39 amostras de leite cru refrigeradas de20 fazendas leiteiras, observando quea média decontagem delevedurasfoi de6,9x 10 U.F.C./ $\mathrm{mL}$ de leite. A contagem defungos no presenteestudo mostrou-se mais elevada que a verificada pelos autores citados.

SABoIs et al. (1991) colheram amostras de leite de tanquederefrigeração del7fazendas. A contagem de leveduras e fungos filamentosos foi $>1.000 / \mathrm{mL}$ de leiteem todas as amostras. Segundo estes autores os altos níveis de fungos nos tanques indicaram uma higieneinsatisfatória duranteo processo deordenha e deficiências do equipamento de ordenha. No presenteestudo, por suavez, observou-seuma mediana de $28,5 \times 10^{2}$ U.F.C. de fungos/ $\mathrm{mL}$ nas amostras de tanque.

N estetrabalho, quando comparadasasmedianas das quantidades deunidades formadoras de colônias defungos/ $\mathrm{mL}$ de leite entreas amostras colhidas de tanques de refrigeração $\left(28,5 \times 10^{2}\right)$, latões de propriedades de exploração leiteira $\left(12,91 \times 10^{2}\right)$, e latões de distribuidores $\left(14,07 \times 10^{2}\right)$, pode-se observar que não houve diferença estatisticamente significante, o que permite concluir que, o nível de contaminação por fungos nas amostras destas três origens foi similar.

Deacordo com a Instrução N ormativa no 51 (BRASIL, 2002), quedispõesobreos regulamentos técnicos de produção, identidade equalidade do leitetipo $A$, do leitetipo $B$, do leite tipo $C$, do leite pasteurizado e do leite cru refrigerado e o regulamento técnico da coletadeleitecru refrigerado eseu transporteagranel, nãoéreferidaacontagem deunidadesformadoras de colônias de fungos/ $\mathrm{mL}$ deleite, havendo somentea contagem padrão em placas (CPP) de unidades formadoras de colônias da totalidade dos microrganismosisolados (bactérias efungos). Com relação a esta última (CPP), a metodologia utilizada para sua realização emprega a temperatura de $32^{\circ} \mathrm{C}$, bem como tempo máximo deincubação de $48 \mathrm{~h}$, fatores estes que poderiam impedir o isolamento de alguns fungos, particularmente, osfilamentososquerequerem maior tempo para crescimento, bem como necessitam temperaturasmais baixas (emtorno de $25^{\circ} \mathrm{C}$ ) paradesenvolvimento. No presente estudo, embora a contagem de unidades formadoras de colônias de fungos/ $\mathrm{mL}$ verificada tenha sido baixa quando comparada com aquelas referidas nas determinações da Instrução N ormativa no 51 para contagem padrão em placas (BRASIL, 2002), deve-se atentar para o risco que constitui a presençadestes microrganismosnoleite, tendo 
em vista que muitos deles são agentes de micoses oportunísticas e/ ou produtores de toxinas, fatores que podem comprometer a qualidade do leite.

Em relação aos transportadores/ distribuidores deleiteinformal, querecolhemoleiteobtidonafazenda eo armazenam em latões dentro derefrigeradores domésticos, deve-seressaltar que, duranteadistribuiçãodesteleite, quepodelevaraté5h, esteémantidoem temperatura ambiente e entregue acondicionado, geralmente, em garrafas de plástico.

A Iguns países estão al ertando as populações sobre os riscos relacionados ao consumo de leite cru, como, por exemplo, osEstadosUnidos. No Estado da Califórnia 34\% da população é de origem hispânica e possui o hábito de consumir leite cru e fabricar queijos frescos como uma prática tradicional ecultural (HEADRICK et al., 1997).

Foram anal isadas amostras de leite provenientes de propriedades leiteiras com grandes diferenças com relação às estruturasfísicas, higienedeordenha edeambiente, manejo esanidadedos animais. Algumasdelasapresentavamsala deordenhaou estábulo com acúmulo de sujidades eanimais aparentemente debilitados, e outras eram altamente tecnificadas, apresentando boa higienedeordenha edeambiente. Emboratenhamapresentadograndes diferenças com relação aos aspectos mencionados, de todas elas foram isoladosfungos leveduriformes dasamostras de leite anal isadas.

Segundo Cousins \& BRAmLey (1981), os microrganismos presentes em amostras de leite provenientes do tanqueder ffrigeração podemter origemapartir de úberes infectados (acometidos por mastite infecciosa), da superfície dos úberes e/ ou dos tetos ou do equipamento deordenha (água delavagem contaminada, manejoinadequadoetc.). Tendo emvistaqueos fungos pertencem ao grupo dos microrganismos ambientais (segundo a classificação dos agentes etiológicos de mastite), a presença destes agentes em amostras detanques de refrigeração, latões defazendas e latões de distribuidores de leite, poderia estar associadaadeficiênciasquanto às condiçõeshigiênicas de obtenção e acondicionamento do produto. Assim sendo, medidas demanejo ad equado frenteàs mastitescausadas por fungos, bem como areal ização apropriada de manejo e higiene de ordenha e de ambiente, poderiam se constituir em fatores capazes deminimizar aocorrênciademastitesporfungosbem como reduzir a contaminação duranteo processo de ordenha.

O presenteestudo permitiu verificar queosfungos estão presentes no leite cru, o que representa um problemaparaoleite, bem como paraseus derivados, pois estes agentes podem causar doenças, bem como podeminterferir comos procedimentos deelaboração de produtos lácteos.
Particularmente, no que tange ao leite cru comercializadodeformainformal, considerando-sea inexistência de qualquer tipo de fiscalização e/ ou controle higiênico-sanitário dos animais que estão envolvidos com a produção destetipo deleite, assim como do processo de obtenção, transporte e comercialização. Os achados deste estudo constituem-seemmotivo depreocupação, principal menteem função do risco representado pelo consumo deste produto sem quetenha sido previamentesubmetido à fervura, particularmente quando considerados indivíduos debilitados ou imunossuprimidos que são os mais susceptíveis às micoses oportunísticas.

\section{REFERÊNCIAS}

AndRAde, M.A. Mastite bovina sub-clínica: prevalência, etiologia e testes de sensibilidade a drogas antimicrobianas. R evistaV et N ews, n.49, p.10-16, 2001.

Araújo, W.N.; Silva, M.H.; Martinez, T.C.N; Banas, S.L.B.; Silveira, V.F. Determinação do número de fungos filamentosos e leveduras no queijo Minas comercializado na região metropolitana deSalvador - Bahia. R evista Brasileira de Saúde e Produção A nimal, v.2, n.1, p.10-14, 2001

BARNET, H.L. \& HUNTER, B.B. Illustrated genera of imperfect fungi. 3.ed., Minneapolis: Burgess Publishing, 1972.

BRANDÃo, S.C.C. Leite: legislação, reponsabilidadeesaúde pública. Revista Balde Branco, v.360, p.68-71, 1994.

BRASIL. Ministério da Agricultura e do Abastecimento. Secretaria de Defesa Agropecuária. Instrução Normativa no51, de18desetembro de2002. A provar os Regulamentos Técnicos de Produção, Identidade eQualidadedo Leitetipo A, do Leitetipo B, do Leite tipo C, do LeitePasteurizado edo LeiteCru Refrigerado eo Regulamento Técnico daColetadeL eiteCru Refrigerado eseu Transportea Granel, em conformidade com os A nexos a esta Instrução Normativa. Publicado no Diário Oficial daU nião de20/ 09/ 2002. Disponível em: \ttp:/ / 200.252.165.21/ das/ dipoa/ in 51b 2002.htm>. A cesso em: 20 out. 2004.

Cousins, C. $\bar{M}$. \& BramLeY, A.J. The microbiology of raw milk. In:Robinson, R.K. (Ed.). D airy microbiology, 1981. v.1, p.119-163.

CRUz, L.C.H. M icologia veterinária. Itaguaí: Imprensa Universitária, 1985. p.191-192.

Desmasures, N.; Opportune, W.; Gueguen, M. Lactococcus spp., yeasts and P seudomonas spp. on teats and udders of milking cows as potential sources of milk contamination. International D airy Journal, v.7, p.643646, 1997.

FonseCa, L.F.L.F. \& SAntos, M.V.Q ualidadedoleitee controle de mastite. São Paulo: Ed. Lemos, 2000. 175p.

HARIDY, M.S.A. Yeast flora of raw milk in El Minia CityEgypt. Cryptogamie-M ycologie, v.13, n.4, p.321-326, 1992.

Headrick, M.L.;Timbo, B.; Klontz, K.C.;Werner, S.B. Profile of raw milk consumers in California. Public Health Reports, v.112, n.5, p.418-422, 1997. 
Jodral, M.; Linan, E.; Acosta, I.; Gallego, C.; Rojas, F.; BENTABOL, A. Mycofloraand toxigenicA spergillusflavus in Spanish milks. International Journal of Food M icrobiology, v.18, p.171-174, 1993.

KERN, M.E. \&BLEVINS, K.S.M icologia médica. 2.ed. SãoPaulo: Premier, 1999. 256p.

Klossowska, A. \& Malinowsi, E. Pathogens in raw milk which affect humans. M edycyna W eterynaryjna, v.57, n.1, p.28-31, 2001.

KreEger-V AN-RIG, N.J.W.T heyeasts: a taxonomic study. 3.ed. A msterdan: Elsevier Science Publisher, 1984. 1082p.

Lacaz, C.S.; Porto, E.; Heing-Vaccari, E.M.; Melo, N.T. Tratado de micologia médica. 9.ed. São Paulo: Sarvier, 2002. $1104 \mathrm{p}$.

LARONE, D.H.M edically important fungi. A guidetoidentification. 3.ed. Washington: ASM Press, 1995. 230p.

LODDER. L. The yeast: a taxonomic study. 2.ed. A msterdam: North Holland Publishing, 1970. 1385p.

Mutukumira, A.N .; Feresu, S.B.;A brahansen, R.K. Chemical and microbiological quality of raw milk produced by smallholder farmersin Zimbabwe.J ournal of Food Protection, v.59, n.9, p.984-987, 1996.
Quelroz, J.C. A valiação sanitária do leite cru distribuído nos M unicípios de J uquitiba el tapecerica da Serra, São Paulo, 1990-1992: estudo experimental. 1995. 75f. Tese(Doutorado) - FaculdadedeSaúdePública, Universidadede São Paulo, São Paulo, 1995.

Sabois, A.; Basilico, J. C.; Simonetta, A. Microbiological quality of raw milk: incidenceof aerobicand anaerobic spore-forming bactéria, yeasts and fungi. Revista A rgentina de Lactologia, v.3, n.5, p.79-90, 1991.

Swinne, D.;Deka, K. E;Assogba, A .;Desmet,P.Identification of yeasts from individual farm tank milk samples in Belgium. Vlaams Diergeneeskundig Tijdschrift, v.66, n.3, p.129-130, 1997.

Trabulsi, L.R.; Alterthum, F.; Gompertz, O. F.; Candeias, J.A.N. M icrobiologia. 3.ed. São Paulo: Atheneu, 1999. 718p.

Von $A R x, J$. A.Thegenera of fungi sporulating in pureculture. 2 ed. Vaduz: Journal Cramer, 1974. 315p.

Recebido em 7/ 4/ 06

A ceito em 31/ 8/ 06 